\title{
Valor Prognóstico da Resolução da Elevação do Segmento ST na Reperfusão Miocárdica
}

\author{
Pablo Balbuena Nery, Luiz Carlos Bodanese, Euler Manenti
}

Porto Alegre, RS

O infarto agudo do miocárdio é a doença mais estudada na história da investigação clínica, sendo que mais de 200 mil pacientes já foram arrolados em estudos multicêntricos de grande escala. Nos Estados Unidos, cerca de 1 milhão de casos de infarto agudo do miocárdio ocorre anualmente ${ }^{1}$.

A terapia de reperfusão tem como objetivo o restabelecimento rápido do fluxo sangüíneo ao miocárdio, cuja função e sobrevivência ficam ameaçadas pela oclusão trombótica da artéria relacionada ao infarto $(\mathrm{ARI})^{2}$.

Dentre as inúmeras modalidades diagnósticas disponíveis, a angiografia é atualmente considerada o padrãoouro para a avaliação da reperfusão. Essa técnica foi primeiramente utilizada por DeWood e cols. ${ }^{2}$, sendo muito empregada, desde então, para a avaliação de pacientes tratados com terapias de reperfusão.

A avaliação angiográfica do fluxo na ARI é realizada segundo a escala qualitativa introduzida pelo Thrombolysis in Myocardial Infarction Study Group (TIMI): grau 0 indica obstrução completa da ARI; grau 1 indica que o contraste penetra além do ponto de obstrução, não opacificando completamente o vaso; grau 2 indica opacificação em todo o vaso, porém com fluxo retardado; grau 3, há perfusão plena na ARI, com fluxo normal ${ }^{3}$.

Com a validação do tratamento endovenoso usando trombolíticos nos estudos clássicos GISSI- ${ }^{4}$ e ISIS- ${ }^{5}$, o próximo passo determinar se uma maior taxa de patência precoce do vaso infartado resultaria em melhor sobrevida. No estudo GUSTO-I, por exemplo, foram analisados angiograficamente 1.210 de um total de 41.021 pacientes $^{6}$. Observouse que pacientes com fluxo TIMI 3 tiveram uma mortalidade de $4,0 \%$ em 30 dias, enquanto pacientes com fluxo TIMI 2 ou TIMI 0-1 uma mortalidade de 7,9\% e 8,6\%, respectivamente.

Recentemente, Gibson e cols. ${ }^{7}$ introduziram um novo método de avaliação angiográfica da perfusão miocárdica

Hospital São Lucas - PUCRS

Correspondência: Euler Manenti - Av. Ipiranga, 6690 - S/300 - 90610-000 - Porto Alegre, RS - E-mail: eulercar@pucrs.br

Recebido para publicação em 2/7/01

Aceito em 22/4/02 em pacientes submetidos à trombólise, o TIMI Myocardial Perfusion Grade (TMP). Essa classificação consiste no refinamento da avaliação angiográfica e estratifica os pacientes em: grau 0 , há opacificação do miocárdio na região irrigada pela ARI; grau 1, o contraste penetra lentamente na microcirculação e permanece até a injeção de contraste subseqüente; o grau 2 indica opacificação do miocárdio e saída lenta do contraste, persistindo até o final da fase de washout, mas não até a próxima injeção; grau 3, há fluxo normal na microcirculação, com presença de mínima quantidade ou ausência de contraste na fase de washout. A mortalidade em 30 dias aumenta quanto menor for o grau do TMP, sendo de $2,0 \%, 4,4 \%, 5,1 \%$ e $6,2 \%$ para TMP graus $3,2,1$ e 0 , respectivamente $(p=0,05)$. Entre os pacientes com fluxo epicárdico TIMI 3, a mortalidade aumenta proporcionalmente à redução da perfusão miocárdica, sendo $0,73 \%$ para TMP grau 3, 2,9\% para TMP grau 2 e 5,0\% para TMP grau $0 / 1(p=0,03)^{7}$.

Van't Hof e cols. ${ }^{8}$ estudaram pacientes tratados com angioplastia coronariana transluminal percutânea primária, utilizando o "grau de opacificação miocárdica" (também chamado de "myocardial blush grade"), análogo ao TMP. No seguimento médio de 1,9 $\pm 1,7$ anos, as taxas de mortalidade foram de $3 \%, 6 \%$ e $23 \%$ para os graus $0 / 1,2$ e 3 , respectivamente $(p<0,0001)$. A “opacificação miocárdica” mostrou-se capaz de subestratificar pacientes com fluxo epicárdico TIMI 3 quanto ao risco de morte em 30 dias. Pacientes com "opacificação miocárdica” grau 0/1, 2 e 3 apresentaram mortalidade de $12,2 \%, 7,4 \%$ e $4,3 \%$, respectivamente 9 .

No entanto, o uso da angiografia apresenta algumas desvantagens, como o fato de ser operador-dependente e representar apenas um momento da anatomia coronariana, e não as flutuações do fluxo coronariano, que ocorrem durante a fase aguda do infarto agudo do miocárdio em 35 $50 \%$ dos pacientes ${ }^{10}$. Além disso, é um método invasivo e caro, não podendo ser utilizado na maioria dos hospitais.

O restabelecimento precoce do fluxo epicárdico não significa sucesso terapêutico, pois cerca de um terço dos pacientes com fluxo normal na ARI não apresenta reperfusão miocárdica tissular, apresentando o fenômeno chamado de 
"no-reflow" 11 . Estudos recentes, testando novos regimes fibrinolíticos e os inibidores plaquetários IIb/IIIa, mostraram uma patência mais rápida ou completa do vaso infartado, mas esse achado não resultou em redução de mortalidade ${ }^{12}$, indicando que a patência normal nos vasos epicárdicos não significa, necessariamente, o restabelecimento da perfusão da microcirculação, objetivo final do tratamento do infarto agudo do miocárdio.

A resolução da elevação do segmento ST (REST) tem sido postulada como uma importante ferramenta na avaliação da reperfusão miocárdica. Assim, os autores buscaram dados que indiquem a aplicabilidade e o valor prognóstico da REST no pós-infarto imediato, bem como sua correlação com a perfusão miocárdica tecidual.

\section{As terapias de reperfusão e a disfunção microvascular}

A isquemia causa dano estrutural ao tecido e à microcirculação miocárdica logo após a trombose coronariana. Uma vez ocorrida a reperfusão miocárdica, desencadeia-se a lesão de reperfusão, causada por infiltração de neutrófilos, geração de radicais livres de oxigênio, ativação do sistema do complemento e moléculas de adesão. Miócitos e arteríolas lesadas, por exemplo, podem reduzir o fluxo da microcirculação, através do aumento da resistência vascular distal, estimulando espasmo arteriolar e causando disfunção endotelial. Além disso, microêmbolos plaquetários desprendem-se após a ruptura da placa, causando a obstrução vascular, que limita ainda mais a perfusão tissular no momento em que a artéria é recanalizada.

A disfunção microvascular também parece ocorrer em artérias não relacionadas ao infarto, possivelmente através de resposta inflamatória global mediada por citocinas e desencadeada pela isquemia miocárdica ${ }^{12}$.

O tempo de isquemia é um forte determinante da disfunção microvascular, propiciando necrose miocárdica e dos capilares centrais da região infartada. Assim, os capilares tornam-se ocluídos por células mortas e debris, perdendo sua integridade anatômica e impedindo a perfusão microvascular. A quantidade de tecido infartado perfundida depende do tempo de isquemia e também de outros fatores, como a extensão da circulação colateral antes da recanalização, a presença de estenose residual na ARI, a extensão da lesão de reperfusão e as condições de enchimento ${ }^{13}$.

A constituição do trombo é particularmente importante no processo de reperfusão. Após a ruptura da placa, é desencadeada a formação de um trombo plaquetário, visto angioscopicamente branco. Cercando rapidamente o núcleo de agregação plaquetária está o trombo vermelho, rico em fibrina. Os trombolíticos - que são melhor definidos como fibrinolíticos - agem sobre a matriz de fibrina, desagregando o trombo vermelho. Assim, a trombina que estava previamente associada à rede de fibrina é liberada, ficando exposta e levando a um estado pró-trombótico. A trombina ativada pode desencadear sua própria formação, além de ser a molécula mais potente na promoção da agregação plaquetária. As plaquetas são resistentes aos fibrinolíticos e, além disso, secretam grandes quantidades de inibidor do ativador do plasminogênio tecidual(PAI-1).

Estudando profundamente o processo de reperfusão, Topol ${ }^{12}$ afirma que os tratamentos fibrinolíticos alcançam apenas a dissolução parcial do trombo, potencializando a embolização distal de partículas na microcirculação.

Certamente, todos esses fatores estão relacionados à disfunção microvascular existente no processo de reperfusão. A avaliação do supradesnível do segmento ST é citada como um marcador do grau de perfusão miocárdica e, conseqüentemente, da eficácia da terapia de reperfusão. Daí a importância de se considerar a REST como uma forma de avaliar a perfusão miocárdica e os riscos de mortalidade a curto e longo prazos.

\section{Resolução da elevação do segmento ST}

Há décadas a REST tem sido recomendada como um marcador útil para a análise da reperfusão à beira do leito. $\mathrm{O}$ impacto clínico do grau de redução da elevação de segmento ST no eletrocardiograma de 12 derivações foi primeiramente estudado por Schröder e cols. ${ }^{14}$, que mostraram mortalidade crescente com a resolução completa $(\geq 70 \%)$, parcial (31 a $69 \%$ ) ou ausente $(\leq 30 \%)$ em pacientes submetidos a regimes fibrinolíticos (fig. 1). Os critérios definidos por Schröder e cols. tiveram sua significância prognóstica validada por outros estudos, sumarizados na tabela $\mathrm{I}^{15-18}$.

Resolução da elevação do segmento ST dentro de 60min após a realização de angioplastia coronariana transluminal percutânea primária com sucesso - fluxo TIMI 3 mostrou-se associada à redução da mortalidade, melhora da função ventricular esquerda e menor incidência de insuficiência cardíaca congestiva, quando comparada à REST incompleta ${ }^{19-23}$. Alguns estudos, com amostras menores de pacientes, consideraram REST completa como $\geq 50 \%$ e ausente como $<50 \%$, também associando a REST completa a um prognóstico melhor.

A REST parece avaliar o fluxo miocárdico tissular, não limitando-se a artéria coronária epicárdica. A ecocardiografia miocárdica contrastada mostra que a REST está associada à perfusão da microvasculatura ${ }^{24}$. Entretanto, estudos realizados possuem amostra com menos de uma centena de pacientes, e a relação direta entre os testes diagnósticos não está estabelecida até o momento.

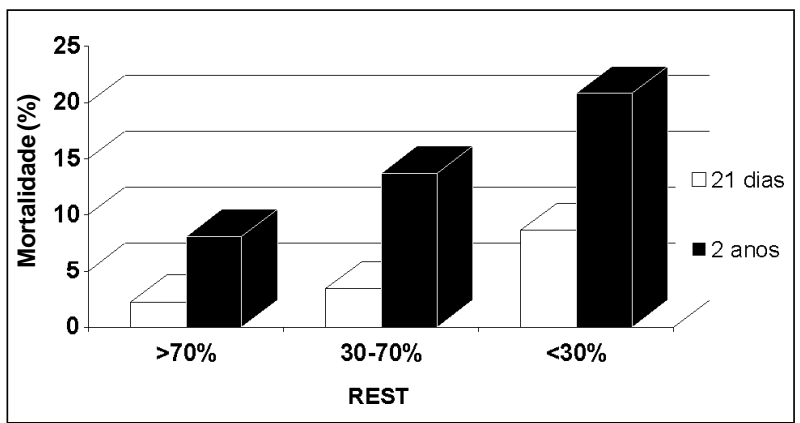

Fig. 1 - Correlação entre resolução da elevação do segmento ST (REST) e mortalidade em 21 dias e 2 anos. 


\begin{tabular}{|c|c|c|c|c|c|c|c|}
\hline Estudo & $\begin{array}{l}\text { Tempo até análise } \\
\text { do ST (min) }\end{array}$ & $\begin{array}{l}\text { Seguimento } \\
\text { (dias) }\end{array}$ & $\mathrm{n}$ & $\begin{array}{l}\text { REST completa } \\
(>70 \%)\end{array}$ & $\begin{array}{l}\text { REST parcial } \\
(30-70 \%)\end{array}$ & $\begin{array}{c}\text { REST ausente } \\
(<30 \%)\end{array}$ & $p$ \\
\hline ISAM $^{14}$ & 180 & 35 & 1.516 & 2,8 & 4,3 & 9,2 & $<0,001$ \\
\hline INJECT $^{15}$ & 180 & 35 & 1.398 & 2,5 & 4,3 & 17,5 & $<0,001$ \\
\hline HIT-4 ${ }^{16}$ & 180 & 35 & 998 & 2,8 & 6 & 14,3 & $<0,001$ \\
\hline TIMI-14 ${ }^{17}$ & 90 & 30 & 444 & 1 & 4,2 & 5,9 & $<0,001$ \\
\hline \multirow[t]{2}{*}{ InTIME-II $^{18}$} & 60 e 90 & 30 & 2.079 & 2,4 & 4,9 & 8,1 & 0,0001 \\
\hline & & 360 & & 3,7 & 7,4 & 10,9 & $<0,0001$ \\
\hline
\end{tabular}

Mais recentemente, em um subestudo do TIMI 14, a mortalidade foi progressivamente maior quanto menor o grau de REST, chegando a menos de $1 \%$ em pacientes com fluxo TIMI 2 e 3 e REST completa ( $\geq 70 \%)$. Pacientes com resolução completa apresentaram uma probabilidade de patência coronariana de $94 \%$ e risco muito baixo de mortalidade a curto prazo. Por outro lado, a ausência de REST não é capaz de predizer com acurácia uma ARI ocluída, sendo que muitos pacientes sem REST apresentavam ARI patente à angiografia. Porém, a ausência de REST foi associada com mortalidade aumentada, mesmo na presença de ARI pérvia ${ }^{17}$. Ainda, a REST $\geq 50 \%$ nos infartos anteriores e $\geq 70 \%$ nos infartos inferiores foi considerado um ponto de corte adequado, pois infartos anteriores estão associados com REST menor do que infartos inferiores ${ }^{17}$. A associação entre a classificação angiográfica e eletrocardiográfica é representada na figura 2 .

Esses achados foram bastante importantes, sendo então realizada a análise de diferentes estratégias de reperfusão utilizando a REST como parâmetro para avaliação da perfusão da microcirculação. Foram analisados 346 pacientes, com mortalidade de apenas $1,1 \%$ nos pacientes com resolução completa da elevação do segmento ST. O uso de inibidores da glicoproteína IIb/IIIa associados ao tPA mostrou maiores taxas de REST completa, quando comparado ao uso isolado do fibrinolítico. Inibidores da glicoproteína IIlb/IIIa não só otimizam a recanalização da artéria culpada como também melhoram a perfusão tecidual ${ }^{25}$.

Desta maneira, o grau de REST após as terapias de reperfusão pode ser considerado um preditor útil e confiável de risco. Entretanto, o eletrocardiograma em pontos estáticos no

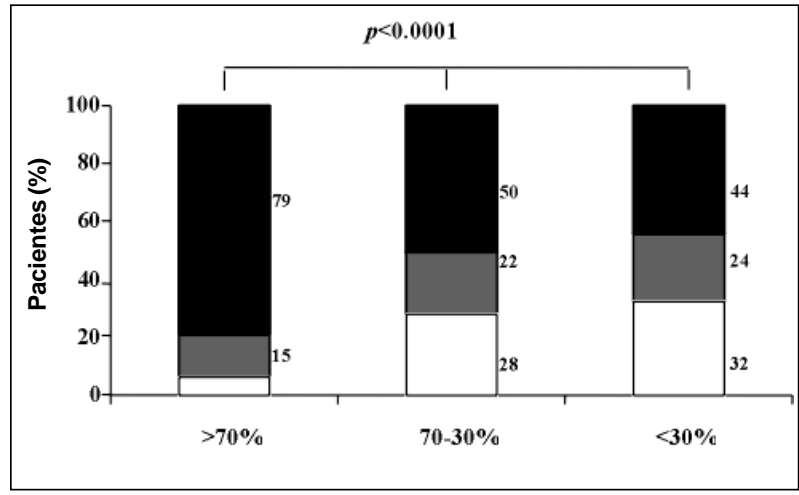

Fig. 2-Classificação de TIMI comparada com a resolução da elevação segmento ST. Área escura, fluxo TIMI3; área hachurada, fluxo TIMI2; área em branco, fluxo TIMI0-1. tempo representa um momento isolado da perfusão tissular. Comojá citado anteriormente, o processo de reperfusãoé dinâmico, sujeito a alterações cíclicas e o momento mais adequado para a análise do segmento ST não está claro ${ }^{26}$.

Vários estudos têm mostrado a capacidade da monitorização contínua do segmento ST na deteç̧ão de falhas na reperfusão, variações cíclicas e estratificação de risco nos pacientes com infarto agudo ${ }^{10,27}$.

O padrão da REST parece estimar o tamanho do infarto e a função ventricular esquerda, permitindo identificar reelevações do segmento ST, o que indica reoclusão e incremento na mortalidade ${ }^{28}$.

Ainda, a monitorização contínua do segmento ST mostrou-se adequada para estimar a incidência de morte intra-hospitalar e insuficiência cardíaca congestiva, condição não atribuída à avaliação angiográfica da reperfusão ${ }^{10}$.

\section{Discussão}

A avaliação da resolução da elevação do supradesnível do segmento ST é capaz de fornecer dados importantes para orientação da terapêutica no infarto agudo do miocárdio. Nesse sentido, a REST se apresenta como uma valiosa ferramenta, uma vez que está associada à melhoria da função ventricular, à redução do tamanho da área de infarto e à maior sobrevida a curto e longo prazos, independente da classificação de TIMI. Pacientes com REST completa têm alta probabilidade de apresentar uma ARI aberta e perfusão miocárdica adequada; portanto, apresentam baixo risco e bom prognóstico.

A ausência de REST não foi capaz de predizer ARI ocluída. Um teste diagnóstico deve ser altamente específico para detectar eventos com probabilidade de ocorrer em cerca de $20 \%$ dos casos, como é caso de uma ARI fechada após os regimes fibrinolíticos atuais ${ }^{6}$. Contudo, é mais lógico que a ausência de REST em pacientes com ARI aberta seja resultado de lesão microvascular, explicando a mortalidade elevada, quando comparados com pacientes com REST completa. Nesses pacientes, o eletrocardiograma parece refletir de maneira mais fidedigna a situação da perfusão miocárdica. Devemos lembrar que embora estudos recentes testando novos regimes fibrinolíticos mostraram patência mais rápida ou completa do vaso infartado, esses não resultaram em redução da mortalidade. A "falha" nesses casos pode estar relacionada ao critério utilizado como objetivo do tratamento.

Pacientes com perfusão miocárdica tissular adequada avaliados pelo TMP e o "grau de opacificação miocárdica" 
evidenciaram redução na mortalidade em proporções bastante semelhantes às observadas em casos de REST completa. Este é mais um dado importante na correlação entre a REST e a avaliação da microcirculação.

Ainda, a REST mostrou-se capaz de estratificar, associada à classificação de TIMI, o risco dos pacientes com fluxo TIMI 2 e 3 , chegando a taxas de mortalidade próximas de $1 \%$ em pacientes com REST completa. Esta capacidade possivelmente está vinculada à avaliação da perfusão miocárdica tecidual.

Ao contrário da angiografia e de outros métodos preconizados para a avaliação da perfusão da microcirculação, o seguimento da REST constitui um método não-invasivo, amplamente difundido, de fácil aplicabilidade e baixo custo, oferecendo excelente relação custo-benefício.

Embora ainda existam lacunas quanto ao tempo ótimo para a realização do eletrocardiograma, os estudos citados utilizaram intervalos que variaram de 60 a 180min. Realização de eletrocardiograma após esse período poderá limitar a eficiência de intervenções de resgate.

Com a vantagem de avaliar a velocidade e a estabilida- de da REST, a monitorização contínua do segmento ST preenche esta lacuna. Em locais que não possuem equipamento para realização de monitorização contínua, certamente o eletrocardiograma seriado é a melhor alternativa disponível.

Faz-se urgente, portanto, a revisão do padrão-ouro para a análise da efetividade da reperfusão no infarto agudo do miocárdio. No presente momento, a resolução da elevação do segmento ST constitui um complemento fundamental à angiografia para avaliação das terapias de reperfusão, permitindo a quantificação mais acurada de risco a curto e longo prazos.

Devido às evidências examinadas, a avaliação da REST deve ser implementada em todos os pacientes tratados com terapias de reperfusão, realizadas através de eletrocardiograma convencional seriado, sempre que a monitorização contínua do segmento ST não estiver disponível. Considera-se resolução completa a redução $\geq 70 \%$ da elevação do segmento ST, a qual estratifica os pacientes como de baixo risco. Podemos também inferir que o valor prognóstico da REST decorre de sua capacidade em avaliar a perfusão da microcirculação miocárdica.

\section{Referências}

1. Braunwald E, Antman EM. Acute myocardial infarction. In: Braunwald E, Zipes DP, Libby P, eds. Heart Disease: A Textbook of Cardiovascular Medicine. $6^{\text {th }}$ Ed. Philadelphia: WB Saunders Co., 2001; Chapter 35: 1114-219.

2. DeWood MA, Spores J, Notske R, et al. Prevalence of total coronary occlusion during the early hours of transmural myocardial infarction. N Engl J Med 1980; 303: 897-902.

3. Vanderschueren S, Van de WerfF. Acute ST-segment elevation myocardial infarction - The open artery and tissue reperfusion. In: Topol EJ, ed. Acute Coronary Syndromes. $2^{\text {nd }}$ Ed. New York: Marcel Dekker, 2001: 173-91.

4. Grupo Italiano per lo Studio della Streptochinasi nell'Infarto Myocardico (GISSI). Effectiveness of intravenous thrombolytic treatment in acute myocardial infarction. Lancet 1986; 1: 397-402.

5. ISIS-2 (Second International Study of Infarct Survival Collaboration Group). Randomized trial of intravenous streptokinase, oral aspirin, both or neither among 17187 cases of acute myocardial infarction. Lancet 1988; 2: 349-60.

6. The GUSTO Angiographic Investigators. The effects of tissue plasminogen activator, streptokinase or both on coronary artery patency, ventricular function and survival after acute myocardial infarction. N Engl J Med 1993; 329: 1615-22.

7. Gibson CM, Cannon CP, Murphy AS, et al. Relationship of TIMI myocardial perfusion grade to mortality after administration of thrombolytic drugs. Circulation 2000; 101: 125-30.

8. Van'tHof AWJ, Liem A, Suryapranata H, et al. Angiographic assessment of myocardial reperfusion in patients treated with primary angioplasty for acute myocardial infarction. Circulation 1998; 97: 2302-6.

9. Stone GW, Lansky AJ, Mehran R, et al. Beyond TIMI-3 flow: The importance of restored myocardial perfusion for survival in high risk patients undergoing primary or rescue PTCA. J Am Coll Cardiol 2000; 35 (2 suppl. A): 403.

10. Shah A, Wagner GS, Granger CB, et al. Prognostic implications of TIMI flow grade in the infarct related artery compared with continuous 12-lead ST-segment resolution analysis. J Am Coll Cardiol 2000; 35: 666-72.

11. Ito $\mathrm{H}$, Okamura $\mathrm{A}$, Iwakura $\mathrm{K}$, et al. Myocardial perfusion patterns related to thrombolysis in myocardial infarction perfusion grades after coronary angioplasty in patients with acute anterior wall myocardial infarction. Circulation 1996; 93: 1993-9.

12. Topol EJ. Acute myocardial infarction: thrombolysis. Heart 2000; 83: 122-6.

13. Agati L. Microvascular integrity after reperfusion therapy. Am Heart J 1999; 138 : S76-78.

14. Schröder R, Dissmann R, Brüggemann T, et al. Extent of early ST segment elevation resolution: a simple but strong predictor of outcome in patients with acute myocardial infarction. J Am Coll Cardiol 1994; 24: 384-91.

15. Schröder R, Wegscheider K, Schröder K, et al. Extent of early ST segment elevation resolution: a strong predictor of outcome in patients with acute myocardial infarction and a sensitive measure to compare thrombolytic regimens: a substudy of the INJECT trial. J Am Coll Cardiol 1995; 26: 1657-64.

16. Schröder R, Zeymer U, Wegscheider K, Neuhaus KL. Comparison of the predictive value of ST-segment elevation resolution at 90 and 180 min after start of streptokinase in acute myocardial infarction: a substudy of the hirudin for improvement of thrombolysis (HIT-4) study. Eur Heart J 1999; 20: 1563-71.

17. De Lemos JA, Antmann EM, Giuliano RP, et al. ST-segment resolution and infarct related artery patency and flow after thrombolytic therapy. Am J Cardiol 2000; 85: 299-304.

18. De Lemos JA, Antman EM, Giuliano RP, et al. Very early risk stratification after thrombolytic therapy with a bedside myoglobin assay and the 12-lead electrocardiogram. Am Heart J 2000; 140: 373-8.

19. Van'tHof AWJ,Liem A, de Boer M,Zijlstra F. Clinical value of 12-lead electrocardiogram after successful reperfusion therapy for acute myocardial infarction. Lancet 1997; 350: 615-9.

20. Santoro GM, Antoniucci D, Valenti R, et al. Rapid reduction of ST-segment elevation after successful direct angioplasty in acute myocardial infarction. Am J Cardiol 1997; 80: 685-9.

21. Matetzky S, Novikov M, Gruberg L, et al. The significance of persistent ST elevation versus early resolution of ST segment elevation after primary PTCA. J Am Coll Cardiol 1999; 34: 1932-8.

22. Claeys MJ, Bosmans J, Veesntra L, et al. Determinants and prognostic implications of persistent ST-segment elevation after primary angioplasty for acute myocardial infarction. Circulation 1999; 99: 1972-7.

23. Matetzky S, Freimark D, Chouraqui P, et al. The distinction between coronary and myocardial reperfusion after thrombolytic therapy by clinical markers of reperfusion. J Am Coll Cardiol 1998; 32: 1326-30.

24. Santoro GM, Valenti R, Buonamici P, et al. Relation between ST-segment changes and myocardial perfusion evaluated by myocardial contrast echocardiography in patients with acute myocardial infarction treated with direct angioplasty. Am J Cardiol 1998; 82: 932-7.

25. De Lemos JA, Antmann EM, Gibson M, et al. Abciximab improves both epicardial flow and myocardial reperfusion in ST-segment elevation myocardial infarction: observations from the TIMI 14 trial. Circulation 2000; 101: 239-43.

26. Roe MT, Ohman EM, Maas ACP, et al. Shifting the open-artery hypotesis downstream: the quest for optimal reperfusion. J Am Coll Cardiol 2001; 37: 9-18.

27. Langer A, KrucoffMW, Klootwijk P, et al. Prognostic significance of ST segment shift early after resolution of ST elevation in patients with myocardial infarction treated with thrmbolytic therapy: the GUSTO-I ST segment monitoring substudy. J Am Coll Cardiol 1998; 31: 783-9.

28. Moons KGM, Klootwijk P, Meij SH, et al. Continuous St-segment monitoring associated with infarct size and left ventricular function in the GUSTO-I trial. Am Heart J 1999; 138: 525-32. 